\title{
SMR
}

\section{Root tip chromosome karyotype analysis of hyacinth cultivars}

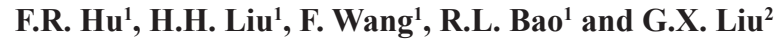 \\ ${ }^{1}$ College of Landscape Architecture of Nanjing Forestry University, \\ Nanjing, China \\ ${ }^{2}$ College of Biology and the Environment of Nanjing Forestry University, \\ Nanjing, China \\ Corresponding author: F.R. Hu \\ E-mail: hufengren@163.com
}

Genet. Mol. Res. 14 (3): 10863-10876 (2015)

Received March 6, 2015

Accepted June 12, 2015

Published September 9, 2015

DOI http://dx.doi.org/10.4238/2015.September.9.24

ABSTRACT. Karyotype analysis in plants helps to reveal the affinity relationships of species and their genetic evolution. The current study aimed to observe chromosome karyotypes and structures of Hyacinthus orientalis. Twenty hyacinth cultivars were introduced from Holland, and their water-cultivated root tips were used as experimental samples. A solution of colchicine $(0.02 \%)$ and 8 -hydroxyquinoline $(0.02 \mathrm{M})$ was used as a 20-h pre-treatment. Subsequently, Carnot I was used for fixation and $45 \%$ acetic acid was used for dissociation. The squash method was selected to prepare chromosome spreads for microscopic observation. The basic chromosome number of the hyacinth cultivar was 8 , and the number of chromosomes in the diploid, triploid, tetraploid, and aneuploid cultivars was $16,23,24,31$, and 32 , respectively. The L-type chromosome was predominant in the chromosomal composition. The hyacinth satellite was located on the short arm in numbers equivalent to the ploidy. This satellite is located on the middle-sized chromosome in the fourth group of chromosomes, demonstrating that Hyacinthus has a more primitive evolution than Lilium and Polygonatum. Among 20 hyacinth cultivars, 'Fondant' had the highest level of evolution and a maximum asymmetric coefficient of $61.69 \%$. Moreover, the ratio 
between the shortest and longest chromosomes in this cultivar was 4.40 , and its karyotype was type $2 \mathrm{C}$. This study may elucidate longterm homonym and synonym phenomena. It may also provide a method of cytological identification as well as direct proof of the high outcross compatibility between hyacinth cultivars.

Key words: Hyacinth; Chromosome; Diploid; Triploid; Tetraploid; Karyotype analysis

\section{INTRODUCTION}

Hyacinthus orientalis, which is known as the daffodil or five-color narcissus, is an herbaceous flower belonging to the hyacinth branch of the Liliaceae family. This species was originally cultivated in the Mediterranean region and in South Africa (Van Sheepen, 1991). In 1562, it was introduced to Eastern Europe from Turkey. The cultivation of $H$. orientalis continuously improved thereafter, and it began to be cultivated in yards. In 1768, more than 2000 varieties of this species were recorded (Darlington et al., 1951). The modern hyacinth is derived from H. orientalis, and its original color was blue and white. In the 18th century, pink, red, purple, and yellow colors were developed (Darlington et al., 1951). Nowadays, the common hyacinth cultivars used for gardening are Holland and Rome species (Shen et al., 2004). Because the famous flowering bulb is planted in the autumn, the hyacinth is widely cultivated in pots and is used as a cut flower worldwide. Its fragrance and graceful style have provided this flower with considerable ornamental value.

The karyotype reflects the chromosome characteristics of various species, helps to reveal their affinity relationships and genetic evolution, and provides reliable cytological evidence for plant system classification and the breeding of new cultivars. Satellites are special structures that are possessed by few chromosomes. Most satellites are located on the short chromosome arm, and the area between the satellite and the short arm of the chromosome is referred to as the secondary constriction or nucleolus organization region (NOR). Stewart (1947) investigated 35 species of Liliaceae based on karyotype analyses and initiated karyotype analyses of the Liliaceae by identifying the NOR based on the residue nucleolar appendages of the mitosis prophase. According to Levitzky (1931) and Stebbins (1971), the main evolutionary trend in the karyotype of flowering plants is reflected by continuously increasing nucleus asymmetry. Shao et al. (1994) found that Liliaceae species display this evolutionary trend. The asymmetry coefficient of Polygonatum species in the verticillata group is clearly larger than that of species with bracteal leaves and Polygonatum species in the alternifolia group, demonstrating that verticillata are more evolutionarily developed.

Research investigating hyacinth chromosomes began in the 1970s. Hyacinth species include diploids with 16 chromosomes; triploids and tetraploids with 24 and 32 chromosomes, respectively; and aneuploids with 23 or 25-31 chromosomes (Darlington et al., 1951; Brat, 1969). Brat examined the chromosomes of 20 cultivars to identify four types of hyacinth chromosomes, i.e., L, Ln, M, and S. Darlington et al. (1951) and Brat (1969) investigated cross breeding of hyacinth cultivars with different ploidy levels. They found that the species with the higher ploidy had a greater capability to crossbred seeds. Due to limitations in their experimental technology, the research of Darlington and Brat cannot be regarded as a true 
karyotype analysis. Since the 1970s, there have been very few reports investigating the chromosomes of hyacinths. Van Tuyl and Toxopeus (1980) described only the chromosome number of 14 hyacinth cultivars in their report on resistance to yellow rotten disease. Hu et al. (2011) reported chromosomal findings for five diploid hyacinths. At present, karyotype analyses of hyacinth chromosomes and their fine structures are in the initial stages.

Because the genetic basis of hyacinths is comparatively narrow due to their origin from one original cultivar and its varieties 'homonym' and 'synonym' have been serious problems since the 17th century (Shen, 2004). In the present study, 20 water-cultivated root-tip hyacinth cultivars were selected as experimental material, and the squash method was used to prepare the chromosome spreads. A microscopy was used to obtain images, and chromosomes with good dispersion were selected for subsequent karyotype analysis. The chromosome karyotype analysis was expected to distinguish different types of chromosomes. The results of this study will provide a reference for analysis of the cross compatibility of hyacinths with different ploidy levels.

\section{MATERIAL AND METHODS}

\section{Materials}

The experimental materials consisted of 20 hyacinth cultivars that were imported from the CBTC International Trade b.v. of Holland. These cultivars included 'Gipsy Queen', 'Purple Sensation', 'Pink Pearl', 'Gipsy Princess', 'Blue Pearl', 'Odysseus', 'Amsterdam', 'Jan Bos', 'City of Haarlem', 'Anna Marie', 'Atlantic', 'Woodstock', 'Amethyst', 'Anna Liza', 'Antarctica', 'Aiolos', 'Peter Stuyvesant', 'Carnegie', 'Fondant', and 'Splendid Cornelia'.

\section{Procedures}

\section{Chromosome preparation}

Experimental materials were selected from water-cultivated root-tip hyacinths. When the root had reached $1 \mathrm{~cm}$ in length, the root tip was cut between 9:00 and 11:00 h. The root tip was then placed in one centrifuge tube that was filled with pre-treatment solution. The solution was prepared by mixing $0.2 \%$ colchicine and $0.002 \mathrm{M} 8$-hydroxyquinoline. After treatment for $20 \mathrm{~h}$, the root tip was transferred to Carnot I solution for fixation for $24 \mathrm{~h}$ (the ratio of absolute ethanol and acetic acid was $3: 1$ ), and was subsequently stored at $4^{\circ} \mathrm{C}$ until use. Ten root tips of various hyacinth cultivars were randomly selected. Acetic acid (45\%) was applied for $2 \mathrm{~h}$ for dissociation, and an alkaline solution was used to compress the red-stained tablet. Finally, the samples were observed and imaged by microscopy using an Olympus $60 *$ objective lens and a DP72 system (Olympus, Japan).

\section{Karyotype analysis}

Five premium-quality images were selected to analyze the chromosome karyotype. The Photoshop CS software (Adobe, USA) was used to cut the chromosomes and match chromosomes from the same source. The Auto CAD2007 software (Autodesk, USA) was used to trace the chromosomes according to their arm curvature, and the Excel software was 
used to calculate the comparative length and arm ratio. SPSS software was used to calculate the average value and standard deviation of five cells.

Chromosome types were classified according to the method described by Levan et al. (1964). The method proposed by Stebbins (1971) and Li (1996) was selected for the karyotype analysis calculations and related parameters. Furthermore, the method proposed by Arano (1963) was used to calculate the asymmetric coefficient of the karyotype.

The related parameters and the formula for the chromosome karyotype analysis have been previously described (Li and Chen, 1985):

Chromosome comparative length $=$ the length of one specific chromosome $\div$ the total length of all of the chromosomes in a chromosome group x $100 \%$.

Chromosome comparative length coefficient $($ IRL) $=$ the length of one specific chromosome $\div$ the average length of all of the groups of chromosomes.

The comparative length of the chromosomes was determined based on the chromosome comparative length coefficient. If the IRL was greater than 1.25 , it was the long chromosome (L). If the IRL was between 1.01 and 1.25, it was the medium-long chromosome (M2). If the IRL was between 0.76 and 1.01, it was the medium-short chromosome (M1). If the IRL was between 0.67 and 0.76 , it was the short chromosome (S).

With reference to the method of karyotype analysis proposed by Li (1996), the length of the satellite was not considered in the calculation of the chromosome length.

Arm ratio $(\mathrm{AR})=$ the length of the long arm $\div$ the length of short arm. This value was used to determine the centric position and karyotype. If AR was 1.00, it was considered metacentric (M). If AR was between 1.01 and 1.70, it was labeled as " $m$ ". If AR was between 1.71 and 3.00, it was labeled as "sm". If AR was between 3.1 and 7.00, it was labeled as "st". If AR was more than 7.01, it was labeled as "t". If AR was $\infty$, it was labeled as "T".

Asymmetric coefficient ASK $(\%)=$ the total arm length of all of the chromosomes :the total length of all of the chromosomes.

Each hyacinth cultivar was associated with one image representing the complete cell karyotype. A previously described method was employed to formulate the karyotype ideograph (Li and Zhang, 1991). Based on the karyotype analysis data, the formula was used to represent the main karyotype feature ( $\mathrm{Li}$ and Zhang, 1991). Based on the ratio between the longest and the shortest chromosome and AR to distinguish the symmetric degree, the karyotypes were classified into 12 types (Stebbins, 1971).

\section{RESULTS}

Ten root tips were colored and squashed for each variety of hyacinth cultivar. For each root tip, more than 30 somatic cells must be observed to determine the quantity of chromosomes and the ploidy levels of the 20 hyacinth cultivars. The results are shown in Table 1.

\section{Diploid hyacinth karyotype analysis}

As shown in Figure 1 and Table 2, the number of chromosomes for six diploids is 16 . 
Table 1. Chromosomes, ploidy levels and colors of hyacinth cultivars.

\begin{tabular}{lccc}
\hline Cultivar & Chromosomes & Ploidy level & Color \\
\hline Gipsy Queen & 16 & $2 \mathrm{x}$ & Orange \\
Purple Sensation & 16 & $2 \mathrm{x}$ & Purple \\
Pink Pearl (2009) & 16 & $2 \mathrm{x}$ & Pink \\
Gipsy Princess & 16 & $2 \mathrm{x}$ & Yellow \\
Blue Pearl & 16 & $2 \mathrm{x}$ & Yellow \\
Odysseus & 16 & $2 \mathrm{x}$ & Orange \\
Amsterdam & 24 & $3 \mathrm{x}$ & Purple \\
Jan Bos & 24 & $3 \mathrm{x}$ & Red \\
City of Haarlem & 23 & $3 \mathrm{x}-1$ & Yellow \\
Anna Marie & 24 & $3 \mathrm{x}$ & Pink \\
Atlantic (2010) & 24 & $3 \mathrm{x}$ & Blue \\
Woodstock & 24 & $3 \mathrm{x}$ & Purple \\
Amethyst & 24 & $3 \mathrm{x}$ & Purple \\
Anna Liza & 24 & $3 \mathrm{x}$ & Purple \\
Antarctica & 24 & $3 \mathrm{x}$ & White \\
Aiolos & 31 & $4 \mathrm{x}-1$ & White \\
Peter Stuyvesant & 32 & $4 \mathrm{x}$ & Blue \\
Carnegie & 31 & $4 \mathrm{x}-1$ & White \\
Fondant & 31 & $4 \mathrm{x}-1$ & Pink \\
Splendid Cornelia & 32 & $4 \mathrm{x}$ & Purple \\
\hline
\end{tabular}

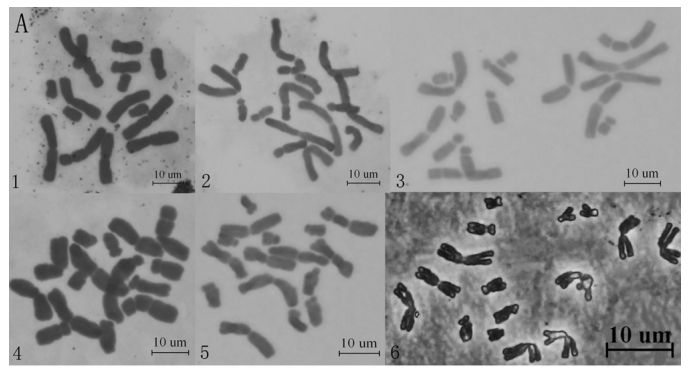

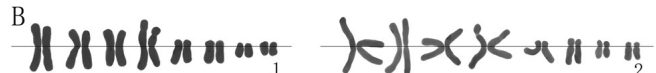

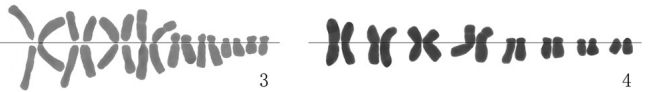

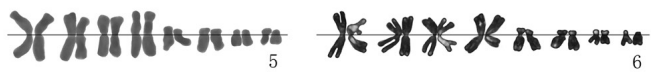
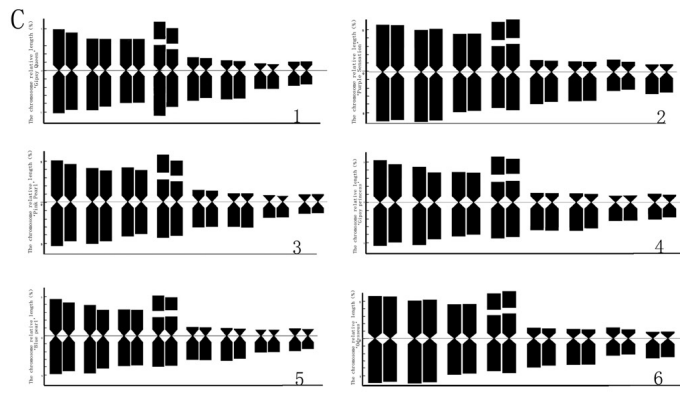

Figure 1. Diploid hyacinth chromosome karyotype and its ideogram. A. and B. chromosome karyotype. C. Ideograms. 1. 'Gipsy Queen'. 2. 'Purple Sensation'. 3. 'Pink Pearl'. 4. 'Gipsy Princess'. 5. 'Blue Pearl'. 6. 'Odysseus'. Scale bar $=10 \mu \mathrm{m}$. 
F.R. Hu et al.

10868

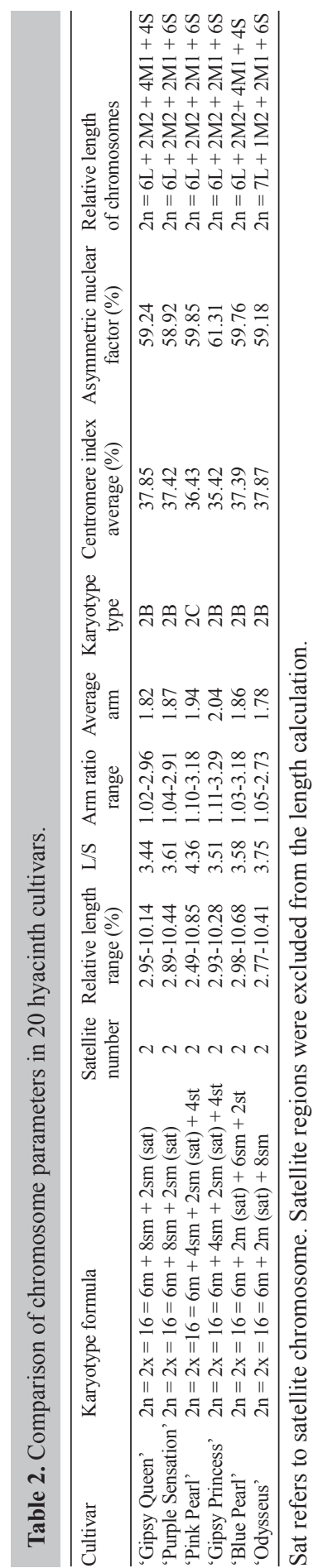

Genetics and Molecular Research 14 (3): 10863-10876 (2015) 
Triploid hyacinth karyotype analysis

As shown in Figure 2 and Table 3, eight cultivars had a chromosome number of 24 (Amsterdam, Jan Bos, Anna Marie, Atlantic, Woodstock, Amethyst, Anna Liza, and Antarctica). One S-type chromosome was missing in City of Haarlem with a quantity of 23, which is a monosomic triploid $(3 \mathrm{X}-1)$.

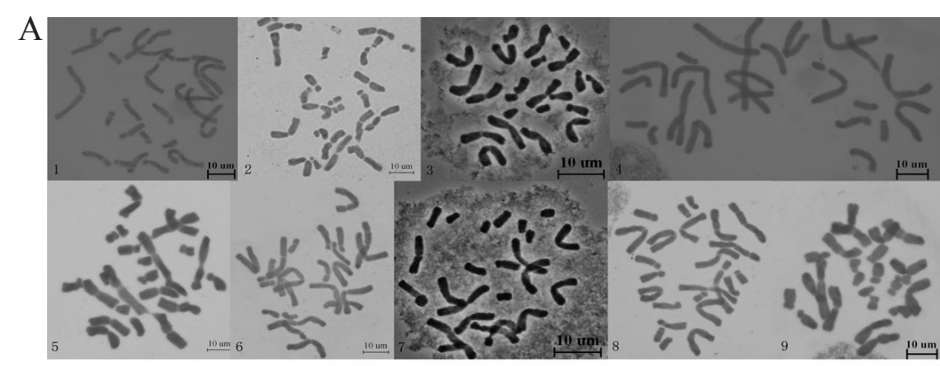

в )

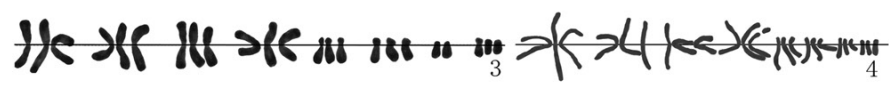

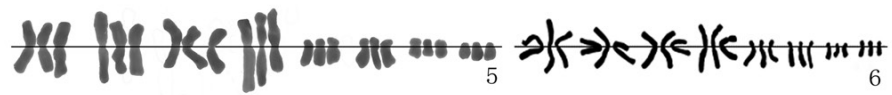

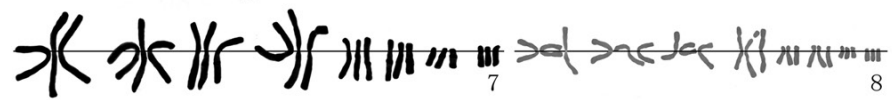

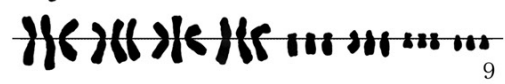

C
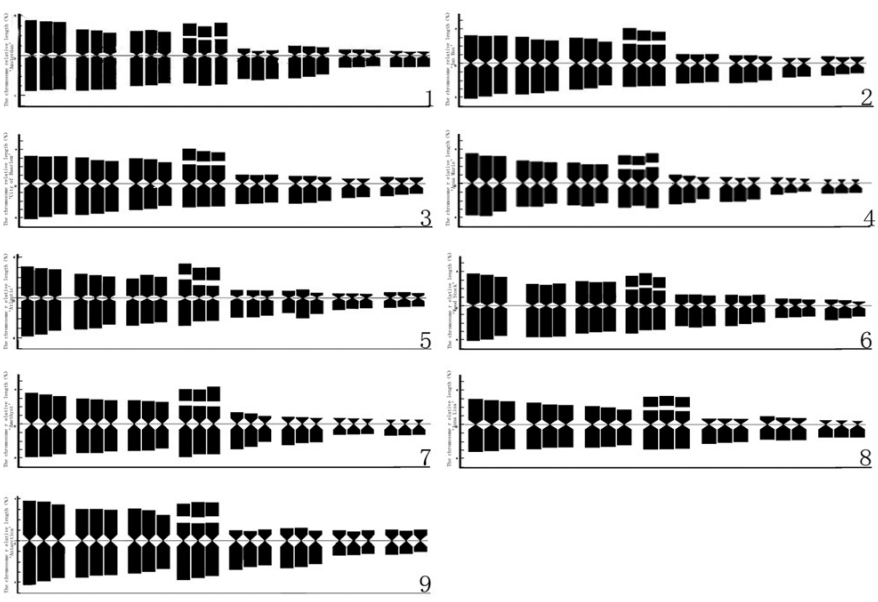

Figure 2. Triploid hyacinth chromosome karyotype and ideogram. A. and B. chromosome karyotypes. C. Ideograms. 1. 'Amsterdam'. 2. 'Jan Bos'. 3. 'City of Haarlem'. 4. 'Anna Marie'. 5. 'Atlantic'. 6. 'Woodstock'. 7. 'Amethyst'. 8. 'Anna Liza'. 9. 'Antarctica'. Scale bar $=10 \mu \mathrm{m}$. 
F.R. Hu et al.

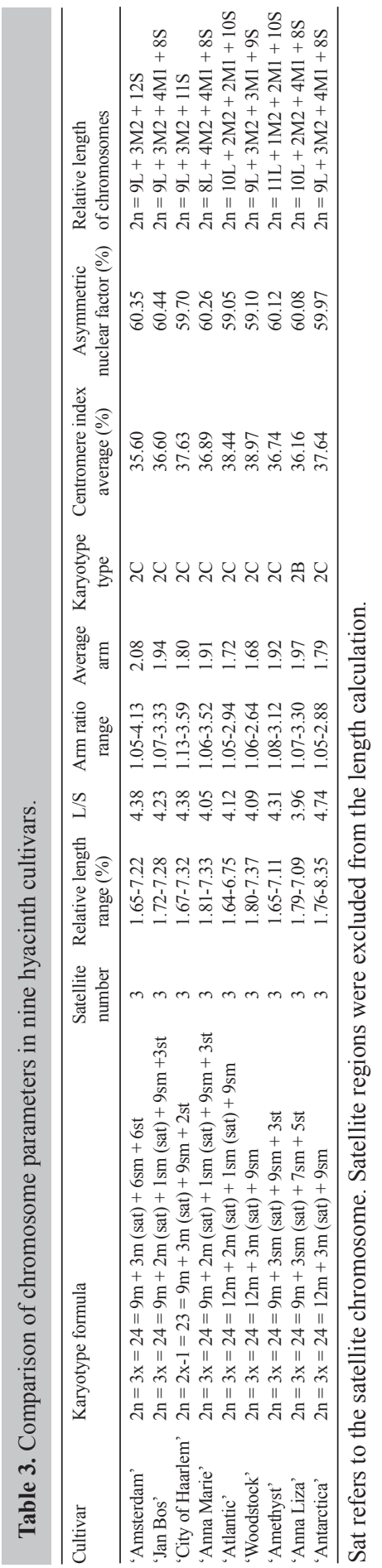




\section{Tetraploid and aneuploid hyacinth karyotype analysis}

As shown in Figure 3 and Table 4, there were 32 chromosomes in Peter Stuyvesant and Splendid. One chromosome was missing in Carnegie and Fondant, which had 31 chromosomes. One S-type chromosome was missing in Aiolos and Carnegie, and one L-type chromosome was missing in Fondant, which is a monosomic tetraploid (4X-1).

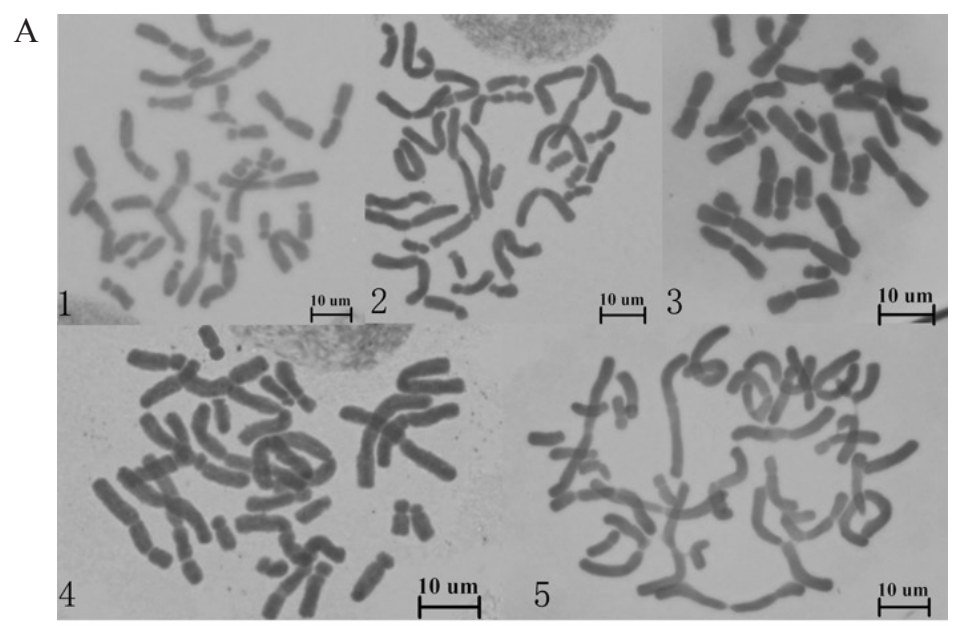

B

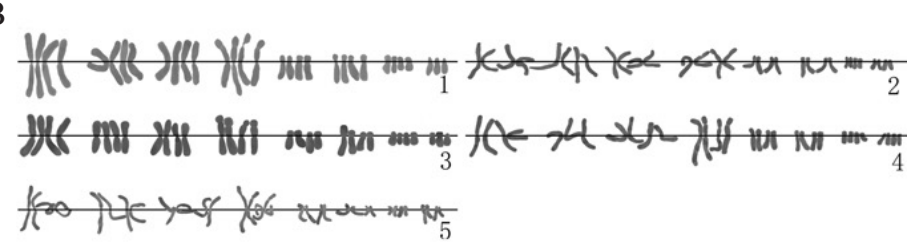

$\mathrm{C}$
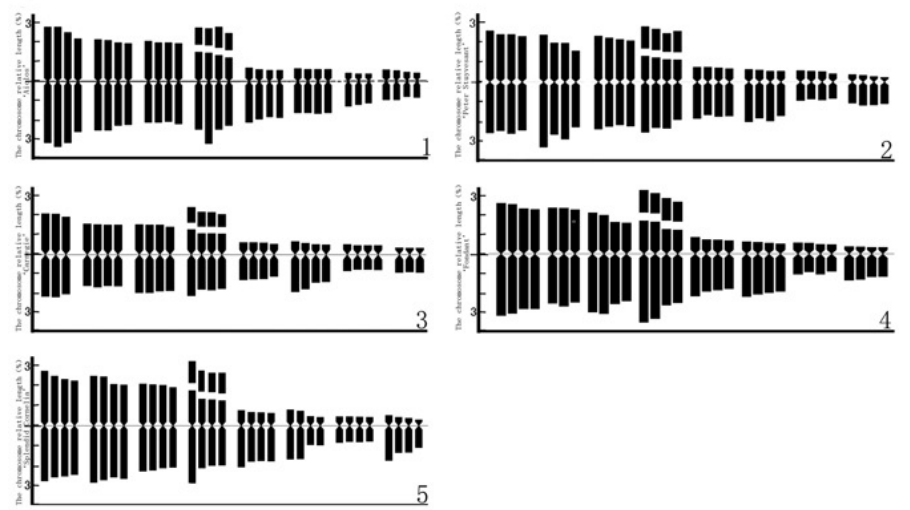

Figure 3. Tetraploid and aneuploid hyacinth chromosome karyotype and ideogram. A. and B. chromosome karyotypes. C. Ideograms. 1. 'Aiolos'. 2. 'Peter Stuyvesant'. 3. 'Carnegie'. 4. 'Fondant'. 5. 'Splendid Cornelia'. Scale bar $=10 \mu \mathrm{m}$. 
F.R. Hu et al.

10872

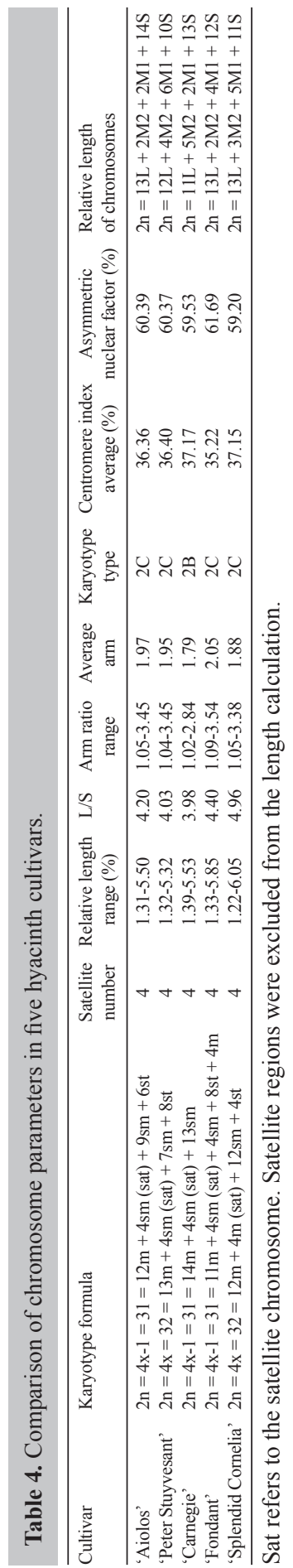

Genetics and Molecular Research 14 (3): 10863-10876 (2015) 


\section{DISCUSSION}

Hyacinths that were cultivated using modern gardening technology have been developed in eastern Mesopotamia, Syria, and Lebanon for six centuries (Shen et al., 2004). Research investigating the hyacinth began before the 1970s. Due to limitations in experimental technology and methods of analysis, previous research has been limited by the quantity and size of the chromosomes (Darlington et al., 1951; Brat, 1969). Analyses of the hyacinth chromosome karyotype and fine structure are currently in their initial stages.

The present study investigated 20 hyacinth cultivars. The basic number of hyacinth chromosomes is eight. Among the 20 cultivars, six were diploid, with a chromosome number of 16 , eight were triploid, with a chromosome number of 24 , two were tetraploid, with a chromosome number of 32, and four were monosomic (chromosome numbers were 23 and 31). This finding is consistent with the results reported by Darlington (1951). Among hyacinth chromosomes, aneuploidy is a common phenomenon. Different hyacinth cultivars showed large variation in chromosome number. Hyacinths with 16-32 chromosomes have previously been cultivated (Brat, 1969). Aneuploid hyacinths can have seeds, which differs from the situation in tulip (Upcott and Philp, 1939). The triploid tulip has a very poor survival capability, and although it may survive, it is also easily aborted.

This study showed that the comparative length of diploid hyacinth ranges from 2.49 to $10.85 \%$. Pink Pearl, Blue Pearl, and Odysseus have a wider range of change. The comparative length of the triploid hyacinth ranges from 1.64 to $8.35 \%$, and Antarctica has the widest range of change. The comparative length of the tetraploid hyacinth ranges from 1.22 to $6.05 \%$. The five hyacinth cultivars have minor differences.

All of the diploid hyacinths have a type 2B karyotype, excluding Pink Pearl, which has $2 \mathrm{C}$. This finding differs from the results reported by $\mathrm{Hu}$ (2011), who stated that Pink Pearl is type $2 \mathrm{~B}$. The underlying reason for this difference may be the length of the satellite used to calculate the total length of the chromosomes. The triploid Anna Liza and tetraploid Carnegie have a type $2 \mathrm{~B}$ karyotype. The other eight triploids with their monomer, and the tetraploid with its monomer have a type $2 \mathrm{C}$ karyotype. Therefore, diploid hyacinths are more primitive than triploid and tetraploid hyacinths.

Darlington et al. (1951) stated that there are five types of hyacinth chromosomes, i.e., L, Ln, M, S1, and S2. The L-type and S-type are not easy to distinguish. Because M-type and S-type chromosomes may be lost easily during meiosis, the L-type chromosome is more stable than the M-type and S-type chromosomes. With reference to the IRL value classification (Kuo, 1972; Li and Zhang, 1991), chromosomes are divided into L, M1, M2, and S (Tables 2-4). By investigating 20 hyacinth cultivars, the results of the present study showed that L-type chromosomes have an absolute advantage. They all include three groups of L-type chromosomes and 2-4 groups of S-type chromosomes (Figures 1-3). This finding is consistent with that reported by Darlington (1951). Regarding aneuploid hyacinths, small chromosomes are most likely to be lost, whereas long chromosomes are not easy to lose (Darlington, 1951). One small chromosome was found to have been lost in City of Haarlem, Aiolos, and Carnegie, resulting in a monomer, whereas one long chromosome was lost in Fondant. This observation confirms the findings of Darlington's research (1951). Darlington (1951) stated that a complete tetraploid hyacinth is very difficult to find. He found that 4M-type and 4S-type chromosomes could not occur in a single hyacinth cultivar. The comparative length, AR, and type of a 
certain group of chromosomes were found to vary significantly with karyotype analysis in hyacinths with $2 n=24$ and $2 n=32$, although they can be matched into complete trivalent and quadrivalent chromosomes.

The comparative length composition of the chromosomes reported by Darlington (1951) and Ved Brat (1967) differ from the findings of the present study. Van Tuyl (1982) stated that Amsterdam has 30 chromosomes and that it is dark pink in color. However, the present study demonstrated that it has 24 chromosomes and is a purple color. Ved Brat (1967) stated that there were 29 chromosomes and three satellite chromosomes. In contrast, in the present study, 31 chromosomes and four satellite chromosomes were detected in Carnegie. The underlying explanation for these differences may be the presence of medium and long chromosomes, which are lost or increased in karyotype analyses; the differences may also be a result of synonymy. Synonymy is a common phenomenon that occurs during the cultivation of hyacinth cultivars. Kersten (1889) reported three types of Queen of the Blues, which were imported from Haarlem, Overveen, and Hilegom. In 1874, Grand Vainqueur displayed white, yellow, and pink colors with a single scape and double scapes. When its karyotype was analyzed, the same cultivar of hyacinth that had been imported in different years was found to have different numbers of chromosomes and ploidy levels.

A satellite is located on the large chromosome, which indicates the later evolution of plants. The hyacinth satellite is located on the 4th middle-sized chromosome. Consequently, the hyacinth has a lower rate of evolutionary change than does Liliaceae and Polygonatum. Darlington (1951) investigated Myosotis, L Innocence and its variation Mme Sophi and found that one of the four satellite chromosomes in the NOR is smaller than the other three normal satellite chromosomes. Hu et al. (2012) found that significant variations are present between the same two sources of satellite chromosomes, although Pink Pearl can be matched to establish eight pairs. When the spread is prepared, one satellite is fairly easy to detach. By analyzing 20 hyacinth cultivars, the present study showed that one or two satellites can be easily detached from the satellite chromosomes, whereas the secondary constriction in satellite chromosomes at rest is too small to be distinguished. This finding is consistent with the results reported by Darlington (1951).

Darlington (1951) investigated the origin of hyacinths based on different numbers of chromosomes. He found that hyacinths with a higher number of chromosomes appeared at a later time. Because hyacinths with different numbers of chromosomes can be cultivated, their evolutionary history may differ from that of flowering bulbs such as the tulip and narcissus. Furthermore, the evolution of hyacinths differs from other gardening plants that have undergone a simple evolution. Diploid hyacinths are more primitive, whereas those with larger numbers of chromosomes are more developed. The hyacinth shares the same evolutionary trend as Polygonatum regarding the number of chromosomes. Aneuploid variables represent the major trend, whereas ploidy levels represent the minor trend, with ascending being major and descending being minor phenomena (Wang et al., 1987; Shao et al., 1994).

Levitzky (1931) and Stebbins (1971) stated that the major evolutionary trend of flowering plants is a continuous increase in nuclear asymmetry. Shao et al. (1994) demonstrated that Liliaceae follows this trend. The present results reveal that the asymmetric coefficient trend has a diploid minimum, triploid medium, and tetraploid maximum value. However, the asymmetric nuclear coefficient for diploid hyacinths reaches $61.31 \%$. Therefore, Gipsy Princess is more developed among the diploid hyacinth cultivars. 
The karyotype is an important index that reflects evolutionary relationships among plants. The present study showed that the other cultivars are type 2B diploid hyacinths, excluding the Pink Pearl, which is $2 \mathrm{C}$. The triploid hyacinths are $2 \mathrm{C}$, excluding Anna Liza, which is $2 \mathrm{~B}$. Thus, diploid hyacinths are more primitive, whereas Pink Pearl has undergone a more developed evolution. As its variability coefficient diminishes, the karyotype becomes more symmetric, showing that the cultivar is more primitive. In the present study, the variability coefficient for diploid and triploid hyacinths reached or exceeded four. This result shows that triploid and tetraploid hyacinths have undergone a more developed evolution. Furthermore, the variability coefficient for the Pink Pearl is 4.36, which confirms that it is one of the more developed cultivars among the diploid hyacinths.

In 1972, hyacinths comprised two color series, i.e., blue and white. Various outcrossing and bud mutations were employed to breed out other color series. White hyacinths are found to be the original variety, whereas blue may belong to one of the recently developed cultivars (Darlington, 1951). Darlington (1951) showed that the color of hyacinths is unrelated to the number of chromosomes, because plants with the same number of chromosomes likely have different colors. In fact, the number of chromosome is related to the evolution of color. Regarding the primitive diploid hyacinths, red color is dominant, whereas blue color accounts for only $15 \%$. Among hyacinths with more than 29 chromosomes, blue color accounts for $80 \%$. Therefore, most of the hyacinths that have been cultivated over the past 100 years are blue. The underlying reason for this phenomenon may be that the blue genes are associated with robust physiological activity and selectivity.

The variability and evolution of plants is a very complicated process. As a method of examining adaptive responses to the environment, karyotype analysis has a useful role in the investigation of the systemic evolution of plants, and it generates highly reliable results (Nie and $\mathrm{Li}, 1993$ ). The present study showed that karyotype analyses can effectively identify ploidy levels and chromosome features in different hyacinth cultivars. In combination with the aneuploid chromosome composition, the species of the hyacinth cultivars can also be determined. Therefore, we postulate that analyses of hyacinths with large numbers of chromosomes and their outcrossing compatibility may provide important information regarding their breeding system. Furthermore, chromosome structure and genetic variability can be ascertained.

\section{Conflicts of interest}

The authors declare no conflict of interest.

\section{ACKNOWLEDGMENTS}

Research supported by the Forestry Science and Technology Promotion Project: Promotion of Excellent Cultivars and Cutting Reproductive Technology of Hyacinthus orientalis [contract \#(2015)20] and the Priority Academic Program Development of Jiangsu Higher Education Institution (PAPD).

\section{REFERENCES}

Arano H (1963). Cytological studies in subfamily Carduoideae (Compositae) of Japan XIII. The karyotype analysis on 
subtribe Gnaphaliineae. Shokubutsugaku Zasshi 76: 419-427.

Brat SV (1969). Fertility and selection in garden Hyacinth II. Zygotic selection. Heredity 24: 189-202.

Darlington CD, Hair JB and Hurcombe R (1951). The history of the garden Hyacinths. Heredity 5: 233-252.

Hu FR, Ren C, Bao RL and Liu GX (2011). Chromosomes analysis of five diploid garden Hyacinth species. Sci. Hortic. 131: 82-87.

Hu FR, Wang F, Bao RL and Xie WR (2012). Pretreatment reagents for hyacinthus root Tip and karyotype analysis. Acta Bot. Boreali-Occidentalia Sin. 32: 2030-2034.

Kersten JH (1889). The cultivation of the Hyacinth in Holland. J. Roy. Hort. Soc. 2: 54-63.

Kuo SR (1972). Karyotype analysis of some Formosan gymnosperms. Tanwania 17: 66-68.

Levan A, Fredga KS and Sandberg AA (1964). Nomenclature for centromeric position on chromosomes. Hereditas 52: 201-220.

Levitzky GA (1931). The karyotype in systematics. Bull. Appl. Bot. Genet. Plant Breed. 27: 220-240.

Li MX and Chen RY (1985). A suggestion on the standardization of karyotype analysis in plants. J. Wuhan. Bot. Res. 4: 297-302.

Li MX and Zhang XF (1991). Plants Chromosome Research Technology. Northeast Forestry University Press, Harbin, 152-153.

Li MX and Zhang ZP (1996). Crop Chromosome and its Research Technology. China University Press, Beijing, 12-37.

Nie RZ and Li MX (1993). Karyotype analysis in cotton plants. Science Publishing House, Beijing, 62-94.

Shao JZ, Zhang DC and Qiang F (1994). Studies on the cytotaxonomy of Polygonatum in Anhui Province. Guihaia 14: 361-368.

Shen Q, Wang H, Zhu HX and Gu JJ (2004). Tulips and Hyacintis. China Forestry Press, Beijing, 63-64.

Stebbins GL (1971). Chromosomal evolution in higher plants. Edward Arnold Ltd., London, 72-123.

Stewart RN (1947). The morphology of somatic chromosomes in Lilium. Am. J. Bot. 34: 9-26.

Upcott M and Philp J (1939). The genetic structure of Tulipa IV. Balance, selection and fertility. J. Genet. 38: 91-123.

Van Sheepen JE (1991). International Checklist for Hyacinths and Miscellaneous Bulbs. Royal General Bulbs Growers' Association (KAVB), Hillegom.

Van Tuyl JM (1982). Breeding for resistance to yellow disease of Hyacinths. II. Influence of flowering time, leaf characters, stomata and chromosome number on the degree of resistance. Euphytica 31: 621-628.

Van Tuyl JM and Toxopeus SJ (1980). Breeding for resistance to yellow disease of Hyacinths. I. Investigation on $\mathrm{F}_{1}$ 's form diallel crosses. Euphytica 29: 555-560.

Ved Brat S (1967). Fertility and selection in garden Hyacinth I. Gametic selection. Heredity 22: 597-601.

Wang JW, Li MX and Li LX (1987) Study on the cytotaxonomy of Polygonatum I. Karyotypes and evolution of eight species. J. Wuhan Bot. Res. 5: 1-10. 